\title{
Primary laryngeal tuberculosis mimicking supraglottic carcinoma
}

\author{
Manigreeva Krishnatreya, Kishore Das, Anupam Sharma, \\ Jagannath D Sharma
}

\begin{abstract}
Introduction: Primary laryngeal tuberculosis is an extremely rare form of tuberculosis and very few have been reported in literature. Case Report: We describe a case of a 35-year-old female who presented with symptoms and sign of a supraglottic carcinoma. The patient presented with progressive hoarseness of two month's duration. Direct fiberoptic laryngoscopy showed a polypoidal growth involving the supraglottic larynx. Tissue sections from the growth showed epithelioid granuloma with Langhans giant cells. Ziehl-Neelsen staining on the tissue section showed the presence of scanty acid-fast bacilli. No primary foci of tuberculosis were detected anywhere else in the body by clinical, radiological and examination of acid-fast bacilli in the bronchioalveolar lavage fluid. Conclusion: Awareness of existence of isolated primary laryngeal tuberculosis is important especially in endemic areas. In case of diagnosis of laryngeal tuberculosis all effort should be made to look for an active or inactive lesion elsewhere in the
\end{abstract}

Manigreeva Krishnatreya ${ }^{1}$, Kishore $\mathrm{Das}^{2}$, Anupam Sharma $^{3}$, Jagannath D Sharma ${ }^{4}$

Affiliations: ${ }^{1}$ MBBS, DLO, Senior Research fellow, Dept of ENT and Head \& Neck Surgery, Dr. B Borooah Cancer Institute, Guwahati, Assam, India; ${ }^{2}$ MBBS, MS, Assistant Professor, Dept of ENT and Head \& Neck Surgery, Dr. B Borooah Cancer Institute, Guwahati, Assam, India; ${ }^{3}$ MBBS, MD, Assistant Professor, Dept of Pathology, Dr. B Borooah Cancer Institute, Guwahati, Assam, India; ${ }^{4}$ MBBS, MD, Professor, Dept of Pathology, Dr. B Borooah Cancer Institute, Guwahati, Assam, India.

Corresponding Author: Dr. Manigreeva Krishnatreya, Room no 32, OPD Block, Dr. B Borooah Cancer Institute, Assam, India 781016; Ph: (+91)9954480612; Email: mani_greeva@yahoo.co.in

Received: 17 January 2013

Accepted: 23 February 2013

Published: 01 July 2013 body before diagnosing as primary laryngeal tuberculosis.

Keywords: Carcinoma, Extra pulmonary tuberculosis, Primary laryngeal tuberculosis

$* * * * * * * * *$

Krishnatreya M, Das K, Sharma A, Sharma JD. Primary laryngeal tuberculosis mimicking supraglottic carcinoma. International Journal of Case Reports and Images 2013;4(7):354-357.

$$
* * * * * * * *
$$

doi:10.5348/ijcri-2013-07-330-4

\section{INTRODUCTION}

Common site for infection with Mycobacterium tuberculosis is lung, but any site may be secondarily or even primarily be involved. Tuberculosis in India is an important disease of public health importance. Recent data have shown a decline in tuberculosis burden of both prevalence and mortality in India [1]. Tuberculosis of the larynx is usually secondary to tuberculosis elsewhere in the body and classically it develops due to direct spread of mycobacterium tubercle bacilli to the larynx from contaminated sputum or due to hematogenous spread. Primary laryngeal tuberculosis is a very rare entity and it results from inhaled tubercle bacilli settling directly on the larynx. Incidence of laryngeal tuberculosis is less than $1 \%$ of all cases of tuberculosis [2].

\section{CASE REPORT}

A 35-year-old female (house wife) presented in the outpatient department of ENT, and head and neck 
surgery with the chief complaints of foreign body sensation of throat for six months and progressive hoarseness of two month's duration. There was no associated history of cough, chest pain, hemoptysis, dyspnea and stridor. The patient was a non-smoker and non-alcoholic. There was also no history of intake of drugs or steroid inhalation.

On general examination, the patient's general condition was good and mild pallor was present. Clinical systemic examination did not reveal any abnormality in the respiratory, cardiovascular and gastro-intestinal system.

On local examination, indirect laryngoscopic examination showed a growth involving the supraglottis. There were no palpable lymph nodes in the neck. Examination of the ears and nose with para nasal sinuses (PNS) did not reveal any abnormality. In the routine examination of blood, hemoglobin estimation was $8.7 \mathrm{~g} / \mathrm{dL}$, erythrocyte sedimentation rate of $15 \mathrm{~mm}$ was seen at the end of first hour (AEFH). Total leucocyte count of 8600 per high powered field and differential leukocyte count of neutrophils $60 \%$, lymphocytes $30 \%$, monocyte $8 \%$ and eosinophil count of $2 \%$ was seen. Liver function tests were normal. Chest $\mathrm{X}$-ray posterior anterior view showed normal lung fields. Direct fiberoptic laryngoscopy examination showed a polypoidal growth with hyperemia involving the epiglottis and bilateral aryepiglottic folds (Figure 1). Bilateral vocal cord mobility was normal. Multiple punch biopsies were taken from the polypoidal growth. Ultrasonography of the abdomen did not reveal any findings suggestive of abdominal tubercular lesion. Serological test for human immunodeficiency virus was non reactive. The histopathological examination showed features of necrotizing inflammatory infiltrate lined by hyperplastic squamous epithelium and epithelioid granuloma with Langhan's giant cells (Figure 2A-B). Ziehl-Neelsen staining on the tissue section showed the presence of scanty acid-fast bacilli. Fiberoptic bronchoscopy was done for collection of bronchioalveolar lavage (BAL) fluid and examination for acid-fast bacilli. On microscopic examination of the bronchioalveolar lavage fluid, acid-fast bacilli were not seen by Ziehl-Neelsen staining. The diagnosis of primary laryngeal tuberculosis was confirmed. The patient was treated with anti-tubercular therapy for six months.

\section{DISCUSSION}

Primary tuberculosis of the larynx is a very rare entity and usually it is secondary to pulmonary tuberculosis. Few cases of primary tuberculosis of larynx have been reported in literature in the recent past $[3,4]$. In the present case, laryngeal tuberculosis is a primary lesion, as no primary foci were detected anywhere else in the body by clinical, radiological including chest X-ray, ultrasonography of the abdomen, Computed tomography (CT) scan of the thorax and examination for acid-fast bacilli in the bronchioalveolar

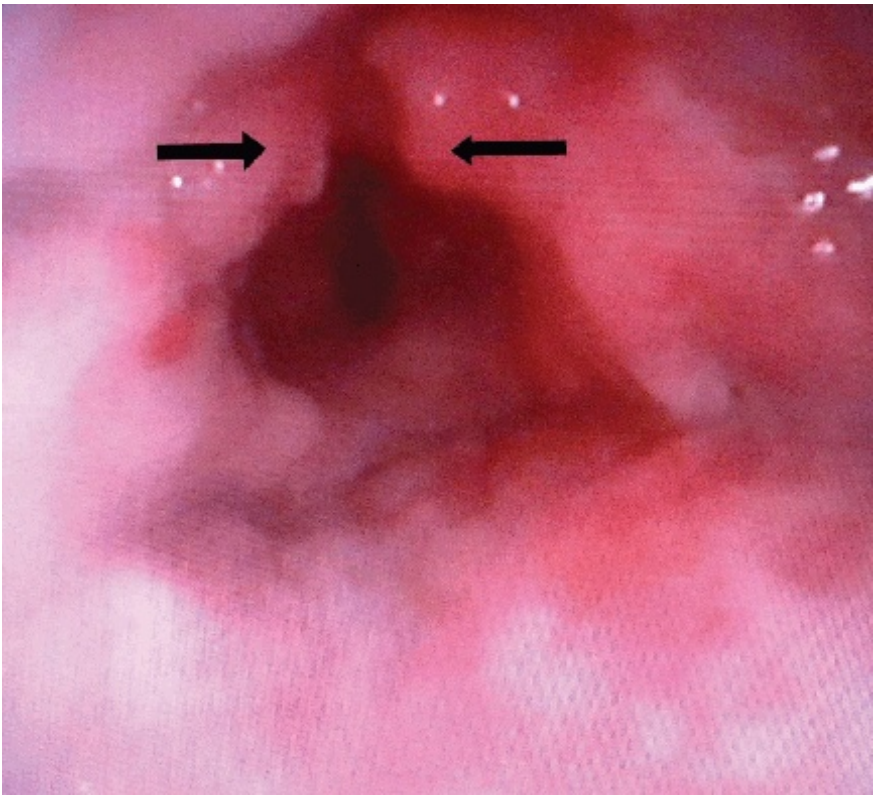

Figure 1: Direct laryngoscopic picture showing growth involving supraglottic larynx with arrows pointing at polypoidal lesion.

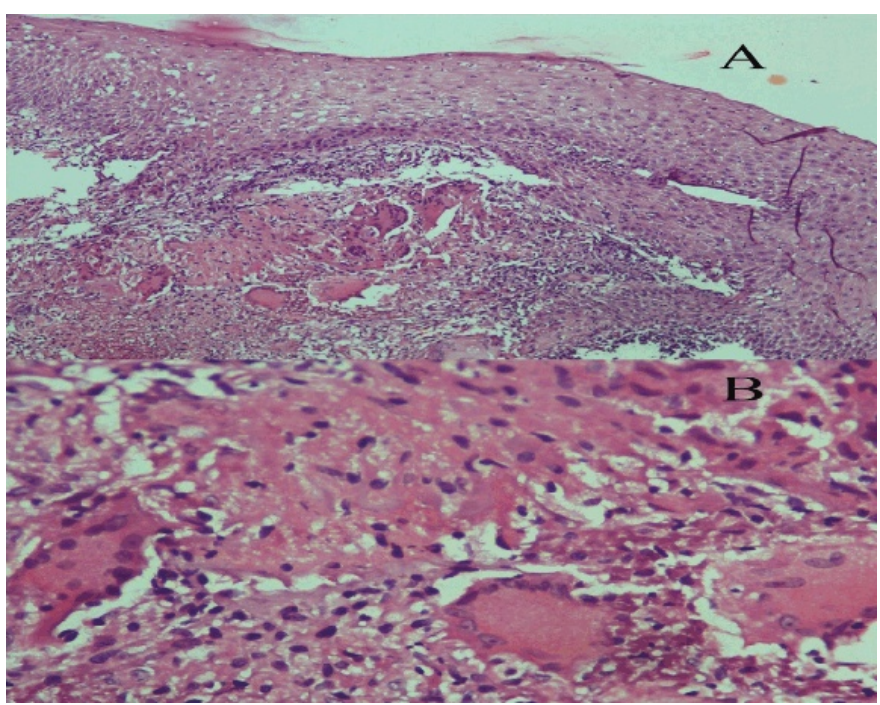

Figure 2: (A) Photomicrograph showing hyperplastic squamous epithelium with underlying epithelioid granuloma with Langhans giant cells and scanty necrosis (H\&E stain, x10), (B) Photomicrograph showing an epithelioid granuloma containing Langhan's giant cells (H\&E stain, x40).

lavage fluid. It has been reported in immune competent individual [5]. In the present case the patient was immune competent. Nishike et al. reported in a series of 15 cases that it commonly affects males more than females and the mean age of presentation is 51 years of age [6]. In the present case the patient is female and age of patient is 35 years, which is an unusual presentation. Laryngeal tuberculosis may present in varying form, from erythematous lesion to ulceration and growth resembling carcinoma [7]. Laryngeal tuberculosis may be categorized into four different types, as proposed by 
Shin et al. These are categorized into the following types-polypoidal in $22.7 \%$, ulcerative in $40.9 \%$, nonspecific in $27.3 \%$ and ulcero-fungative growth in $9.1 \%$ of laryngeal tuberculosis cases [8]. In the present case it presented as a polypoidal growth. It should be always borne in mind that tuberculosis and carcinoma may coexist in the same patient, so complete work-up for malignancy must be carried out before diagnosing as laryngeal tuberculosis without associated carcinoma. Laryngeal tuberculosis is the most common polypoidal lesion of larynx and laryngeal tuberculosis is the most common ENT manifestation of tuberculosis [9]. Earlier, it was seen that laryngeal tuberculosis frequently involves the posterior part of larynx, particularly in bed ridden patients and in whom sputum got collected in the inter arytenoid region. Fernandes et al. reports the most common part to be involved is the vocal cord (50-70\%), and least affected is the epiglottis [10]. In this case the involved site is the epiglottis and bilateral aryepiglottic folds (supraglottic larynx) and the patient had progressive hoarseness of two month's duration and there was laryngoscopic finding of a growth involving the supraglottis, this led to the suspicion of a supraglottic carcinoma. Patients presenting with hoarseness without any pulmonary symptoms in endemic regions, laryngeal tuberculosis should be considered in the differential diagnosis. The response to anti-tubercular treatment for laryngeal tuberculosis is remarkable. Patients with less severe form of extra pulmonary tuberculosis like laryngeal tuberculosis; a six-month treatment with anti-tubercular therapy is sufficient [11]. There was complete remission of symptoms and signs of the patient on follow-up after six months from starting of the anti-tubercular therapy. Direct laryngoscopic examination and biopsy from the laryngeal lesion is mandatory to establish a definitive diagnosis. Histopathological examination can differentiate between a tuberculosis and malignancy.

\section{CONCLUSION}

Awareness of existence of isolated primary laryngeal tuberculosis is important especially in endemic areas. In case of diagnosis of laryngeal tuberculosis all efforts including clinical, radiological and examination for acid-fast bacilli in the bronchoalveolar lavage should be made to look for an active or inactive lesion elsewhere in the body before diagnosing as primary laryngeal tuberculosis.

$* * * * * * * * *$

\section{Author Contributions}

Manigreeva Krishnatreya - Substantial contributions to conception and design, Acquisition of data, Analysis and interpretation of data, Drafting the article, Final approval of the version to be published

Kishore Das - Substantial contributions to conception and design, Drafting the article, Final approval of the version to be published
Anupam Sharma - Substantial contributions to conception and design, Drafting the article, Final approval of the version to be published

Jagannath D Sharma - Substantial contributions to conception and design, Revising it critically for important intellectual content, Final approval of the version to be published

\section{Guarantor}

The corresponding author is the guarantor of submission.

\section{Conflict of Interest}

Authors declare no conflict of interest.

\section{Copyright}

(C) Manigreeva Krishnatreya et al. 2013; This article is distributed under the terms of Creative Commons attribution 3.0 License which permits unrestricted use, distribution and reproduction in any means provided the original authors and original publisher are properly credited. (Please see www.ijcasereportsandimages.com /copyright-policy.php for more information.)

\section{REFERENCES}

1. Sachdeva KS, Kumar A, Dewan P, Kumar A, Satyanarayan S. New Vision for Revised National Tuberculosis Control Programme (RNTCP): Universal access - "Reaching the un-reached". Indian J Med Res 2012;135(5):690-4.

2. Egeli E, Oghan F, Alper M, Harputlouglu U, Bulut I. Epiglottic tuberculosis in a patient treated with steroids for Addison's disease. Tohoku J Exp Med 2003;201(2):119-25.

3. Baxi S, Jha S. Primary laryngeal tuberculosis a rare entity. J Indian Med Assoc 2010;108(3):178-9.

4. Edizer DT, Karaman E, Mercan H, Alimoglu Y, Esen T, Cansiz H. Primary tuberculosis involving epiglottis: a rare case report. Dysphagia 2010;25(3):258-60.

5. Sá LC, Meirelles RC, Atherino CC, Fernandes JR, Ferraz FR. Laryngo-pharyngeal Tuberculosis. Braz J Otorhinolaryngol 2007;73(6):862-6.

6. Nishiike $\mathrm{S}$, Irifune $\mathrm{M}$, Doi $\mathrm{K}$, Sawada $\mathrm{T}$, Kubo $\mathrm{T}$. Laryngeal tuberculosis; a report of 15 cases. Ann Otol Rhinol Laryngol 2002;111(10):916-8.

7. Lindell MM jr, Jing BS, Wallace S. Laryngeal tuberculosis. AJR Am J Roentgenol 1977;129(4):677-80.

8. Shin JE, Nam SY, Yoo SJ, Kim SY. Changing trends in clinical manifestation of laryngeal tuberculosis. Laryngoscope 2000;110(11):1950-3.

9. Kulkarni NS, Gopal GS, Ghaisas SG, Gupte NA. Epidemiological considerations and clinical features of ENT tuberculosis. $\mathrm{J}$ Laryngol Otol 2001;115(7):555-8.

10. Fernandes L, Mesqnita A. Stridor presentation in laryngeal tuberculosis. Indian J Tuberc 1997;44:93-4.

11. Sharma SK, Mohan A. Extrapulmonary tuberculosis. Indian J Med Res 2004;120(4):316-53. 
Access PDF of article on other devices

other devices

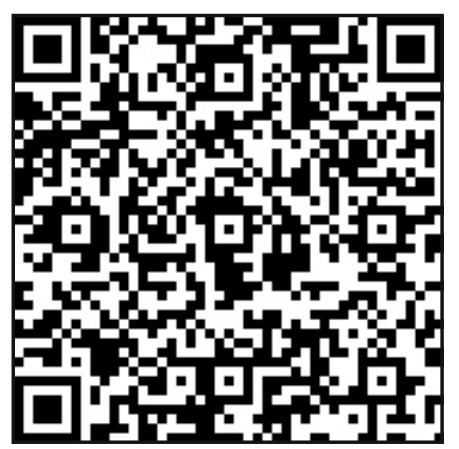

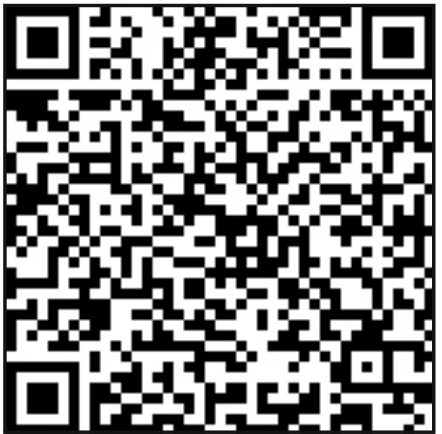

